\title{
Tangential Winding Coil Probes \\ for Dipole, Quadrupole and \\ Sextupole Magnet Measurements
}

\section{Introduction}

The number of coils for a rotating coil probe of "tangential winding geometry" depends on the requirements of the magnef measurements. In order to measure the main field, multipole coefficients and the location of the magnetic center axis, two sets of coils are sufficient for a rotating coil probe of "radial winding geometry." A probe of tangential winding geometry, on the other hand, requires more than two sets of coils to measure the above field parameters. This note describes probes of tangential winding geometry with a minimum number of coils for dipole, quadrupole and sextupole magnet measurements.

\section{Two-dimensional Magnetic Field}

The 2-D field in the aperture of a magnet may be expressed as

$$
B_{y}+i B_{x}=B_{0} \sum_{m=0}^{\infty}\left(b_{m}+i a_{m}\right)(x+i y)^{m},
$$

or

$$
B_{\theta}+i B_{I}=\sum_{n=1}^{\infty} C_{n} \exp \left(-i n \alpha_{n}\right)\left(\frac{z}{R}\right)^{n-1} \exp (i \theta) \text {, }
$$

where $z=x+l y=r \exp (i \theta)$ and $R$ is a reference radius. The multipole coefficients $C_{n}, b_{n}$ and $a_{n}$ are related to each other as

$$
\frac{C_{n}}{R^{n-1}} \exp \left(-i n \alpha_{n}\right)=B_{0}\left(b_{n-1}+i a_{n-1}\right)
$$

In Eq. (1) it is defined that $b_{0}=1.0$ for dipole, $b_{1}=1.0 \mathrm{~cm}^{-1}$ for quadrupole and $b_{2}=1.0 \mathrm{~cm}^{-2}$ for sextupole magnets. 


\section{DISCLAIMER}

This report was prepared as an account of work sponsored by an agency of the United States Government. Neither the United States Government nor any agency thereof, nor any of their employees, make any warranty, express or implied, or assumes any legal liability or responsibility for the accuracy, completeness, or usefulness of any information, apparatus, product, or process disclosed, or represents that its use would not infringe privately owned rights. Reference herein to any specific commercial product, process, or service by trade name, trademark, manufacturer, or otherwise does not necessarily constitute or imply its endorsement, recommendation, or favoring by the United States Government or any agency thereof. The views and opinions of authors expressed herein do not necessarily state or reflect those of the United States Government or any agency thereof. 


\section{DISCLAIMER}

Portions of this document may be illegible in electronic image products. Images are produced from the best available original document. 


\section{Dipole Magnet Probe}

Figure 1 shows the cross section of a probe configuration for dipole measurements. It consists of two coil sets: one $\Delta$-coil with an opening angle of $\Delta$ and one $\pi$-coil (a dipole coil with an opening angle $\pi$ ).

The flux linkage of the two coils at an angular position of $\theta$ is given by

$$
\begin{aligned}
\phi_{\Delta}(\theta)-\phi_{D}(\theta)= & \operatorname{LN}_{\Delta} \sum_{n=1}^{\infty} C_{n}\left(\frac{r}{R}\right)^{n-1} \frac{r}{n} 2 \sin n\left(\theta-\alpha_{n}\right) * \\
& {\left[\sin \left(\frac{n \Delta}{2}\right)-\left(N_{D} / N_{\Delta}\right) \sin \left(\frac{n \pi}{2}\right)\right], }
\end{aligned}
$$

where $\phi_{\Delta}$ and $\phi_{D}$ are flux linkages of the $\Delta$-coil and $\pi$-coil respectively, $L$ the effective magnetic length of the measuring magnet, and $N_{\Delta}$ and $N_{D}$ are the number of turns of the $\Delta$-coil and $\pi$-coil.

The condition for the bucking of the dipole field component is

$$
\mathrm{N}_{\Delta} \sin \left(\frac{\Delta}{2}\right)-\mathrm{N}_{D}=0
$$

With a choice of the probe coil parameters, $N_{\Delta}=14, N_{D}=3$ and $\Delta=24.74725^{\circ}$, relative sensitivities of multipole coefficients are listed in Table 1 .

\section{Quadrupole Magnet Probe}

Figure 2 shows the cross section of a probe configuration for quadrupole magnet measurements. The probe consists of one $\Delta$-coil, one $\pi$-coil and two $\pi / 2$-coils (quadrupole coils).

The flux linkage of the probe at an angle $\theta$ is given by

$$
\begin{aligned}
& \phi_{\Delta}(\theta)-\phi_{D}(\theta)-\phi_{Q}(\theta)=I_{\Delta} \sum_{n=1}^{\infty} C_{n}\left(\frac{r}{R}\right)^{n-1} \frac{r}{n} 2 \sin n\left(\theta-\alpha_{n}\right) * \\
& {\left[\sin \left(\frac{n \Delta}{2}\right)-\left(N_{D} / N_{\Delta}\right) \sin \left(\frac{n \pi}{2}\right)-\left\{1+(-1)^{n}\right\}\left(N_{Q} / N_{\Delta}\right) \sin \left(\frac{n \pi}{4}\right)\right],}
\end{aligned}
$$

where $\phi_{Q}$ is the flux linkage of the two quadrupole coils each with $N_{Q}$ turns. 
The bucking conditions of the dipole and quadrupole fields components of the probe coil in Eq. (6) are

$$
\begin{aligned}
& C_{1}\left[N_{\Delta} \sin \left(\frac{\Delta}{2}\right)-N_{D}\right]=0, \\
& C_{2}\left[N_{\Delta} \sin (\Delta)-2 N_{Q}\right]=0 .
\end{aligned}
$$

With a choice of the probe coll parameters, $N_{\Delta}=18, N_{D}=3, N_{Q}=3$, and $\Delta=19.47122^{\circ}$, relative sensitivities of multipole coefficients are listed in Table 2.

The implication of Eq. (7) is further discussed for the case when the rotating axis of the probe is displaced from the magnetic axis of the measuring magnet. When the rotating coil and magnet axes do not coincide as shown in Figure 3, the multipole coefficients with respect to the $x y$-coordinate system and those with respect to the $x^{\prime} y^{\prime}$-coordinate system are related as

$$
\begin{aligned}
& \sum_{J=1}^{\infty} C_{J^{\prime}} \exp \left(-i J \alpha^{\prime}{ }_{J}\right)\left(\frac{Z^{\prime}}{R}\right)^{J-1} \\
& =\sum_{J=1}^{\infty} \sum_{N=J}^{\infty} C_{N} \exp \left(-i N \alpha_{N}\right) \frac{(N-1) !}{(N-J) !(J-1) !}\left(\frac{Z^{\prime}}{R}\right)^{J-1}\left(\frac{Z}{R}\right)^{N-J} .
\end{aligned}
$$

From Eq. (9) the field in the $x^{\prime} y^{\prime}$-coordinates for a quadrupole magnet $C_{n}$ $(n \neq 2)<<C_{2}$ is given by

$$
B_{\theta}^{\prime}+i B_{x}^{\prime}=C_{2} \exp \left(-i 2 \alpha_{2}\right) \frac{z_{o}+z^{\prime}}{R} \exp \left(i \theta^{\prime}\right) \text {. }
$$

The flux linkages of the coils in Fig. 2 due to Eq. (10) are

$$
\begin{aligned}
& \phi_{\Delta}\left(\theta^{\prime}\right)=\operatorname{LN}_{\Delta} \frac{C_{2}}{R}\left[r_{0} r 2 \sin \left(\theta^{\prime}+\theta_{0}-2 \alpha_{2}\right) \sin \left(\frac{\Delta}{2}\right)\right. \\
& \left.\quad+r^{2} \sin 2\left(\theta^{\prime}-\alpha_{2}\right) \sin (\Delta)\right], \\
& \phi_{D}\left(\theta^{\prime}\right)=\operatorname{LN}_{D} \frac{C_{2}}{R} x_{0} r 2 \sin \left(\theta^{\prime}+\theta_{0}-2 \alpha_{2}\right), \\
& \phi_{Q}\left(\theta^{\prime}\right)=\operatorname{LN}_{Q} \frac{C_{2}}{R} 2 x^{2} \sin 2\left(\theta^{\prime}-\alpha_{2}\right) .
\end{aligned}
$$


Flux linkage, $\phi \Delta\left(\theta^{\prime}\right)-\phi_{D}\left(\theta^{\prime}\right)-\phi_{Q}\left(\theta^{\prime}\right)$ of Eqs. (11), (12) and (13) satisfies the bucking conditions of Eqs. (7) and (8). In this case the dipole coefficient in Eq. (7) is equivalent to $C_{1}=C_{2} r_{0} / R$.

As shown in Eq. (13) the flux linkage of the two $\pi / 2$-coils does not depend on $r_{0} \exp \left(i \theta_{0}\right) ;$ it depends only on the quadrupole field strength of the magnet.

In summary, the quadrupole magnet probe has the following features.

1. In the measurements of multipole coefficients, the dipole and quadrupole components are cancelled out, whether the probe axis is located in the magnetic axis or not.

2. The detection of the magnetic center axis is expressed in Eq. (12). When the radil of the winding conductors of the $\pi$-coil differ by $\Delta r$, an additional term due to the quadrupole field,

$$
\mathrm{IN}_{D} \frac{\mathrm{C}_{2}}{\mathrm{R}} \Delta \mathrm{r} \cdot \mathrm{r} \sin 2\left(\theta^{\prime}-\alpha_{2}\right)
$$

should be added to Eq. (12).

3. The quadrupole field integral is measured from the two $\pi / 2$-coils as shown in Eq. (13).

\section{Sextupole Magnet Probe}

Figure 4 shows the cross section of a probe configuration for sextupole magnet measurements. The probe consists of one $\Delta$-coil, two $\pi / 2$-coils and two $\pi / 3$-coils.

The coils are connected to have the flux linkage of the coils such that

$$
\begin{aligned}
& \phi_{\Delta}(\theta)-\phi_{Q}(\theta)-\phi_{S}(\theta)={ }^{L N}{ }_{\Delta} \sum_{n=1}^{\infty} C_{n}\left(\frac{r}{R}\right)^{n-1} \frac{r}{n} 2 \sin n\left(\theta-\alpha_{n}\right) * \\
& {\left[\sin \left(\frac{n \Delta}{2}\right)-\left\{1+(-1)^{n}\right\} N_{Q} / N_{\Delta} \sin \left(\frac{n \pi}{4}\right)-\left\{1-(-1)^{n}\right\} N_{S} / N_{\Delta} \sin \left(\frac{n \pi}{6}\right)\right],}
\end{aligned}
$$

where $\phi_{S}(\theta)$ is the $\pi / 3$-coil flux linkage and $N_{S}$ is the number of turns for each of the $\pi / 3$-coils. 
The bucking conditions for the quadrupole and sextupole field components in Eq. (14) are

$$
\begin{aligned}
& \mathrm{C}_{2}\left[\mathrm{~N}_{\Delta} \sin (\Delta)-2 \mathrm{~N}_{Q}\right]=0 \\
& \mathrm{C}_{3}\left[\mathrm{~N}_{\Delta} \sin \left(\frac{3 \Delta}{2}\right)-2 \mathrm{~N}_{S}\right]=0 .
\end{aligned}
$$

With a choice of the probe parameters $N_{\Delta}=15, N_{Q}=2, N_{S}=3$ and $\Delta=15.71879^{\circ}$, relative sensitivities of the multipole coefficients are listed in Table 3.

When the probe axis is displaced from the magnetic axis as shown in Fig. 3, for a sextupole magnet $C_{n}(n \neq 3)<<C_{3}$, one obtains from Eq. (9)

$$
B_{\theta}^{\prime}+i B_{I}^{\prime}=C_{3} \exp \left(-i 3 \alpha_{3}\right)\left(\frac{z_{0}+Z^{\prime}}{R}\right)^{2} \exp \left(i \theta^{\prime}\right) \text {. }
$$

The flux linkages of the coils in Fig. 4 due to Eq. (17) are

$$
\begin{aligned}
& \phi_{\Delta}\left(\theta^{\prime}\right)=\operatorname{LN}_{\Delta} \frac{C_{3}}{R^{2}}\left[2 x_{0} x^{2} \sin \left(2 \theta^{\prime}+\theta_{0}-3 \alpha_{3}\right) \sin (\Delta)\right. \\
&\left.+\frac{2}{3} x^{3} \sin 3\left(\theta^{\prime}-\alpha_{3}\right) \sin \left(\frac{3 \Delta}{2}\right)\right], \\
& \phi_{Q}\left(\theta^{\prime}\right)=\operatorname{LN}_{Q} \frac{C_{3}}{R^{2}} 4 r r^{2} \sin \left(2 \theta^{\prime}+\theta_{0}-3 \alpha_{3}\right), \\
& \phi_{S}\left(\theta^{\prime}\right)=\operatorname{IN}_{S} \frac{C_{3}}{R^{2}} \frac{4}{3} x^{3} \sin 3\left(\theta^{\prime}-\alpha_{3}\right) .
\end{aligned}
$$

The flux linkage, $\phi_{\Delta}\left(\theta^{\prime}\right)-\phi_{Q}\left(\theta^{\prime}\right)-\phi_{S}\left(\theta^{\prime}\right)$ of Eqs. (18), (19), and (20) also satisfies the bucking conditions of Eqs. (15) and (16). The quadrupole coefficient in Eq. (15) is equivglent to $2 r_{0} C_{3} / R$. The dipole field term in Eq. (17) which is proportional to $\mathrm{r}_{0}{ }^{2} \mathrm{r}$, is neglected in the subsequent calculations in Eqs. (18) (20).

From Eqs. (19) and (20) the magnetic axis and sextupole field integral are measured. 
Table 1. Probe Coil Parameters for Dipole Magnets.

$\mathrm{N}_{\Delta}=14, \mathrm{~N}_{\mathrm{D}}=3$, and $\Delta=24.74725^{\circ}$.

\begin{tabular}{|c|c|r|r|}
\hline & \multicolumn{1}{|c|}{ A } & \multicolumn{1}{c|}{ B } & \multicolumn{1}{c|}{ C } \\
\hline 1 & Tangential coil probe lor dipole magnet measurements \\
\hline 2 & & 14 & \\
\hline 3 & ndelta & 3 & \\
\hline 4 & ndipole & 24.74725 & \\
\hline 5 & delta & 3.14159265 & \\
\hline 6 & pi & & \\
\hline 7 & & & \\
\hline 8 & & Sensitivity & \\
\hline 9 & n & $-2.22181 E-09$ & \\
\hline 10 & 2 & 0.418616148 & \\
\hline 11 & 3 & 0.817784251 & \\
\hline 12 & 0.76034362 & \\
\hline 13 & 4 & 0.66757898 & \\
\hline 14 & 5 & 0.962416139 & \\
\hline 15 & 6 & 1.212541421 & \\
\hline 16 & 7 & 0.987718359 & \\
\hline 17 & 8 & 0.71700792 & \\
\hline 18 & 9 & 0.831602913 & \\
\hline 19 & 10 & 0.907563346 & \\
\hline 20 & 11 & 0.522744093 & \\
\hline 21 & 12 & 0.113639211 & \\
\hline 22 & 13 & 0.117871036 & \\
\hline 23 & 14 & & \\
\hline & & & \\
\hline
\end{tabular}

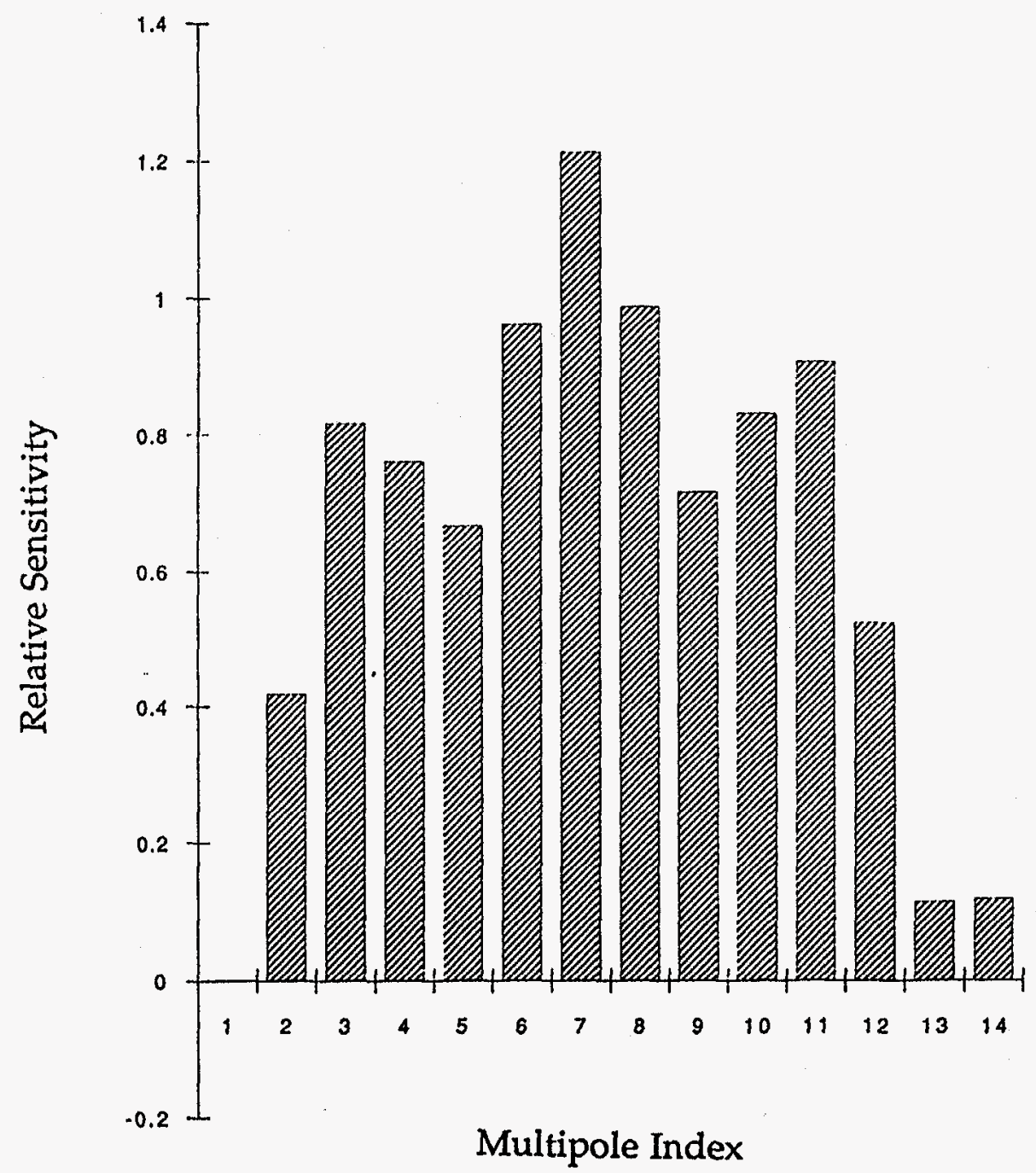


Table 2. Probe Coil Parameters for Quadrupole Magnets.

$\mathrm{N}_{\Delta}=18, \mathrm{~N}_{\mathrm{D}}=3, \mathrm{~N}_{\mathrm{Q}}=3$, and $\Delta=19.47122^{\circ}$.

\begin{tabular}{|c|c|c|c|}
\hline & A & B & C \\
\hline 1 & Tangential co & probe for quadrupole mag & net measurements \\
\hline \multicolumn{4}{|c|}{ 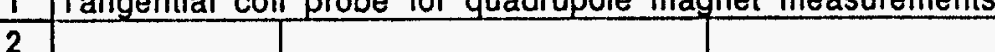 } \\
\hline 3 & ndelta & 18 & \\
\hline 4 & ndipole & 3 & \\
\hline 5 & nquad & 3 & \\
\hline 6 & delta & 19.47122 & \\
\hline 7 & $\mathrm{pi}$ & 3.14159265 & \\
\hline \multicolumn{4}{|l|}{8} \\
\hline 9 & & & \\
\hline 10 & n & sensitivity & \\
\hline 11 & 1 & 0.002435306 & \\
\hline 12 & 2 & $-1.1405 \mathrm{E}-08$ & \\
\hline 13 & 3 & 0.654630379 & \\
\hline 14 & 4 & 0.628539343 & \\
\hline 15 & 5 & 0.584344565 & \\
\hline 16 & 6 & 1.185185165 & \\
\hline 17 & 7 & 1.094823318 & \\
\hline 18 & 8 & 0.97772789 & \\
\hline 19 & 9 & 0.832471075 & \\
\hline 20 & 10 & 0.658436218 & \\
\hline 21 & 11 & 1.122502216 & \\
\hline 22 & 12 & 0.892370729 & \\
\hline 23 & 13 & 0.636536396 & \\
\hline 24 & 14 & 1.024234164 & \\
\hline 25 & 15 & 0.725365342 & \\
\hline
\end{tabular}

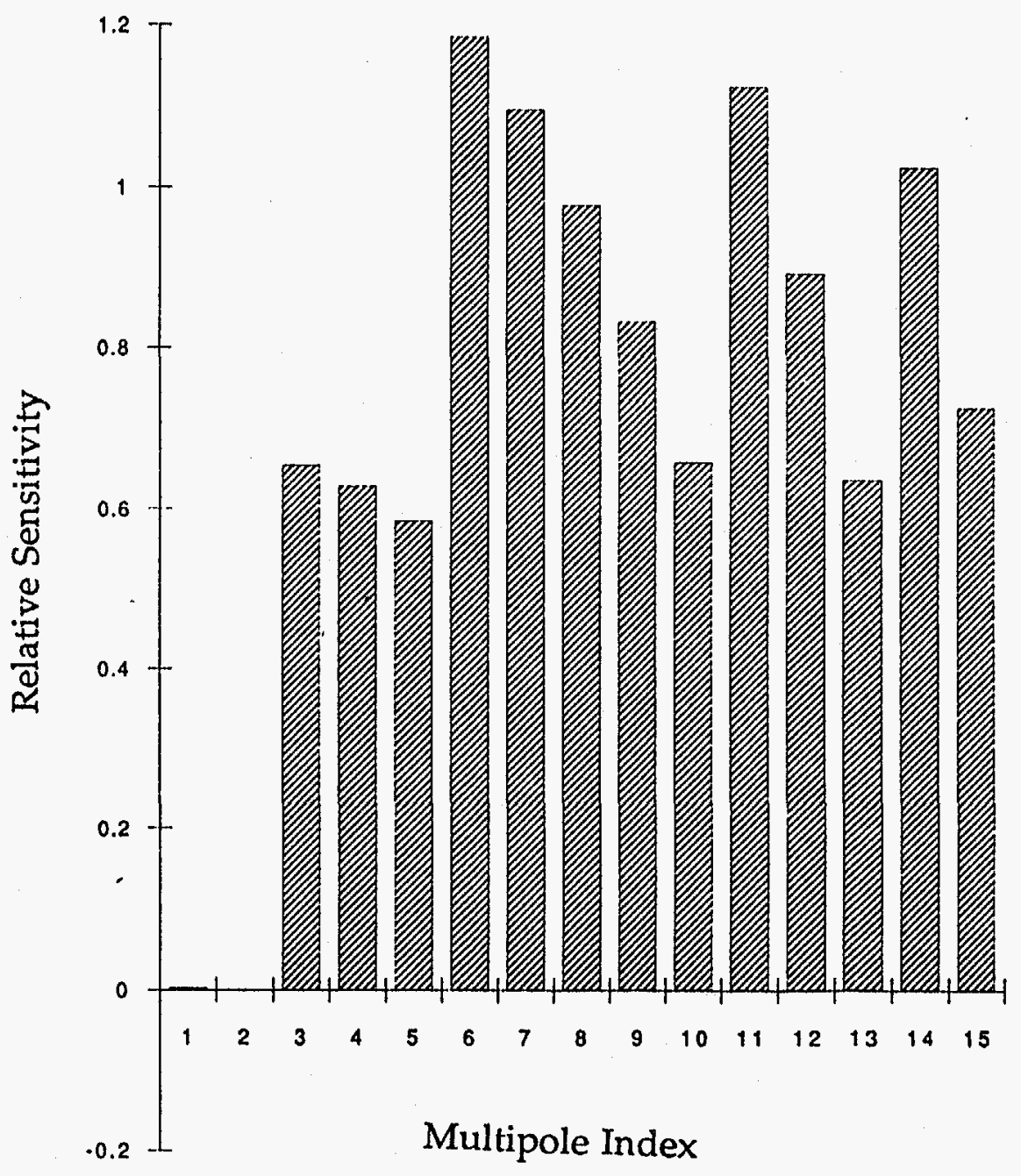


Table 3. Probe Coil Parameters for Sextupole Magnets.

$N_{\Delta}=15, N_{Q}=2, N_{S}=3$, and $\Delta=15.7187^{\circ}$.

\begin{tabular}{|c|c|c|c|}
\hline & $\mathbf{A}$ & B & $\mathrm{C}$ \\
\hline 1 & \multicolumn{3}{|c|}{ Tangential coil probe for sextupole magnet measurements } \\
\hline 2 & & & \\
\hline 3 & ndelta & 15 & \\
\hline 4 & nquad & 2 & \\
\hline 5 & nsext & 3 & \\
\hline 6 & delta & 15.71879 & \\
\hline 7 & $\mathrm{pi}$ & 3.14159265 & \\
\hline 8 & & & \\
\hline 9 & & & \\
\hline 10 & $n$ & sensitivity & \\
\hline 11 & 1 & -0.063257453 & \\
\hline 12 & 2 & 0.004249477 & \\
\hline 13 & $\overline{3}$ & $1.03893 E-07$ & \\
\hline$\overline{14}$ & $\overline{4}$ & 0.521569358 & \\
\hline 15 & 5 & 0.433340014 & \\
\hline 16 & 6 & 0.999878932 & \\
\hline 17 & 7 & 1.019309831 & \\
\hline 18 & 8 & 0.890015225 & \\
\hline 19 & 9 & 1.344000112 & \\
\hline 20 & 10 & 0.713583631 & \\
\hline 21 & 11 & 1.198084759 & \\
\hline 22 & 12 & 0.997168434 & \\
\hline 23 & 13 & 0.777518557 & \\
\hline 24 & $\overline{14}$ & 1.206170931 & \\
\hline 25 & 15 & 0.483839735 & \\
\hline
\end{tabular}

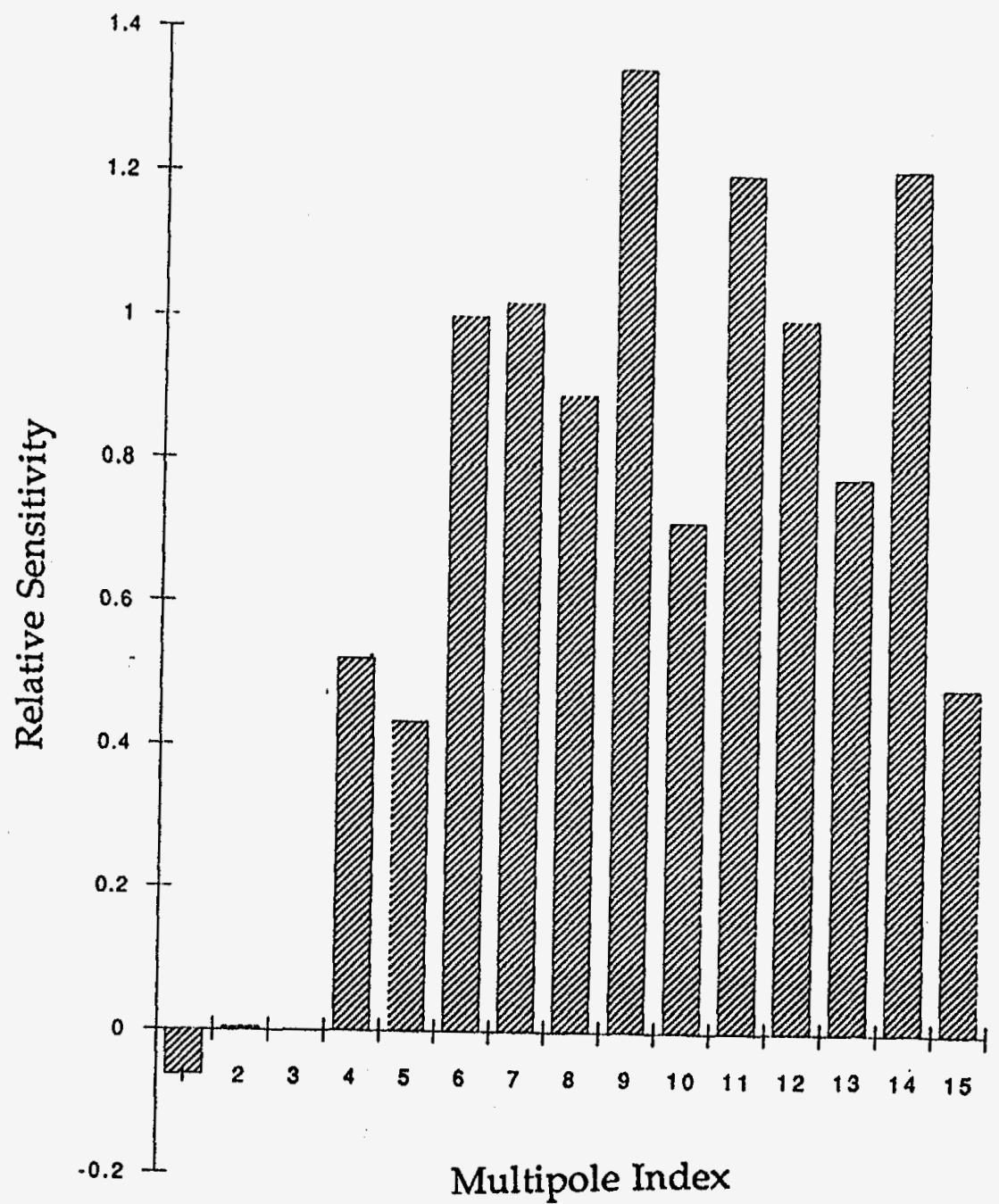




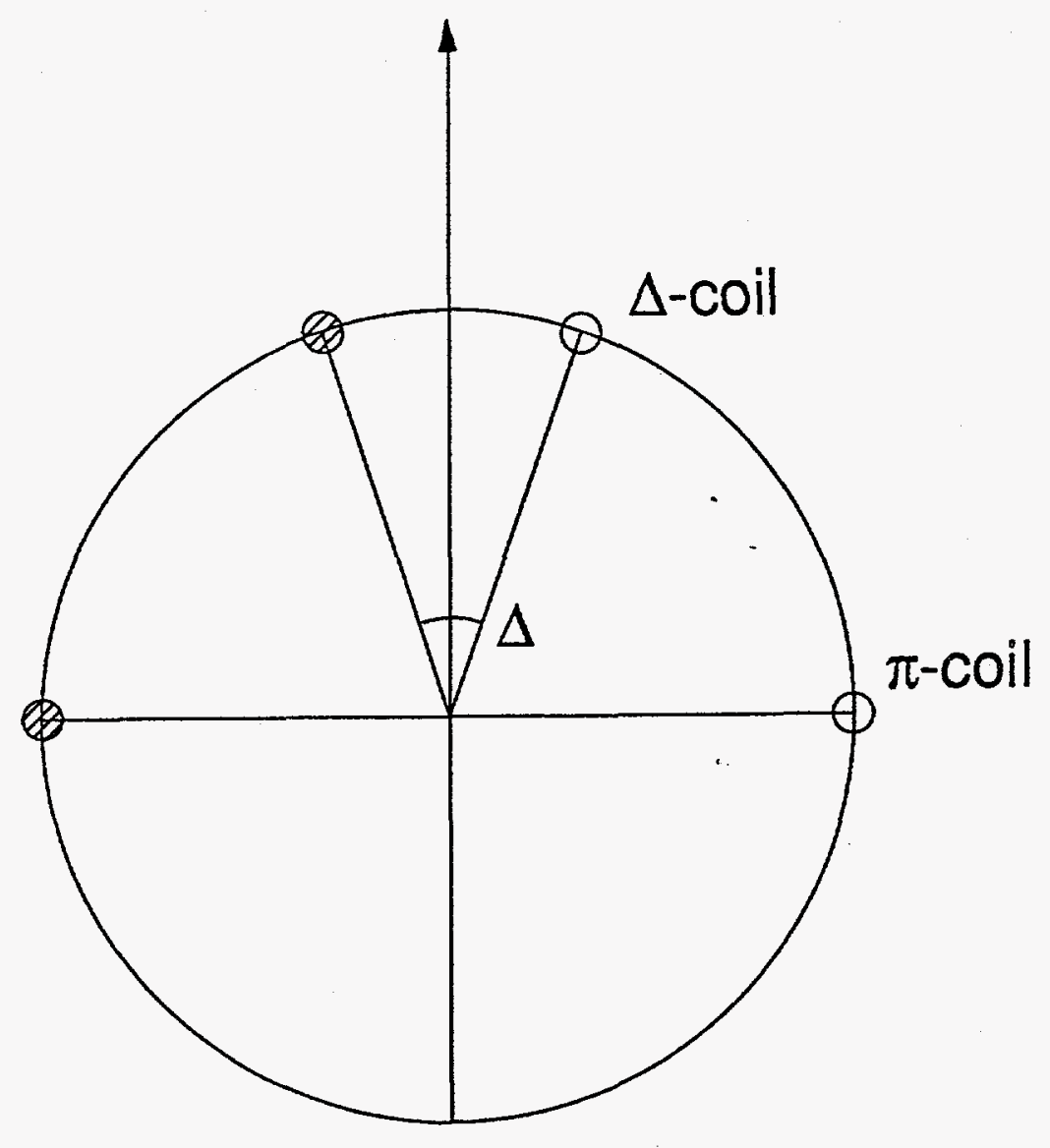

Fig. 1. Cross section of a tangential wing coil for dipole magnet measurements. It consists of one $\Delta$-coil and one $\pi$-coil (dipole coil) which is used for the bucking of the dipole field and for the measurements of dipole field integral. The vertical axis, which bisects the angle of the $\Delta$-coil is the reference angular direction, $\theta=0$. 


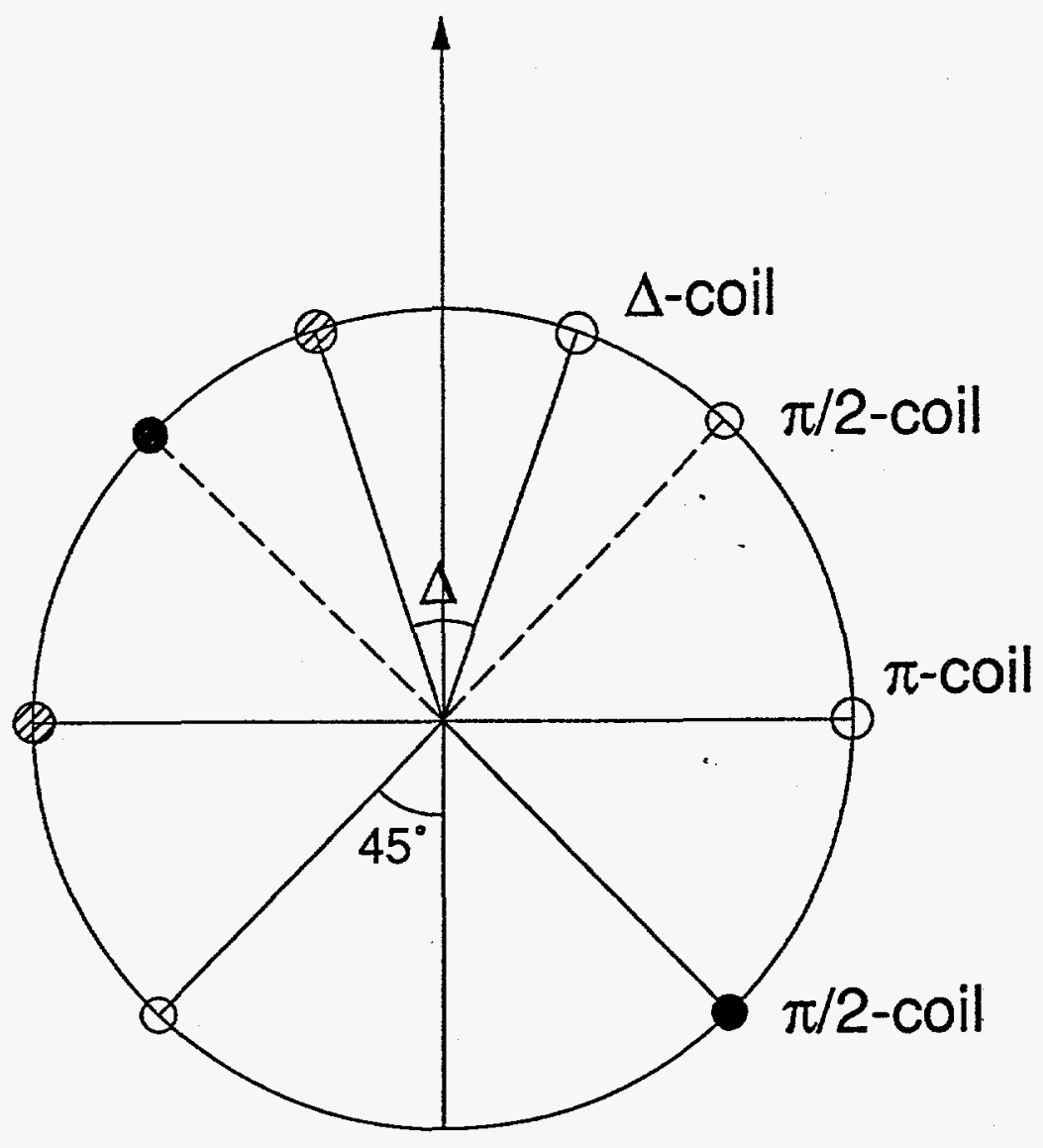

Fig. 2. Cross section of a tangential winding coil for quadrupole magnet measurements. It consists of one $\Delta$-coil, one $\pi$-coil which is used for the bucking of the dipole field and for the measurements of the magnetic center, and two $\pi / 2$-coils for the bucking of the quadrupole field and for the measurements of quadrupole field integral. 


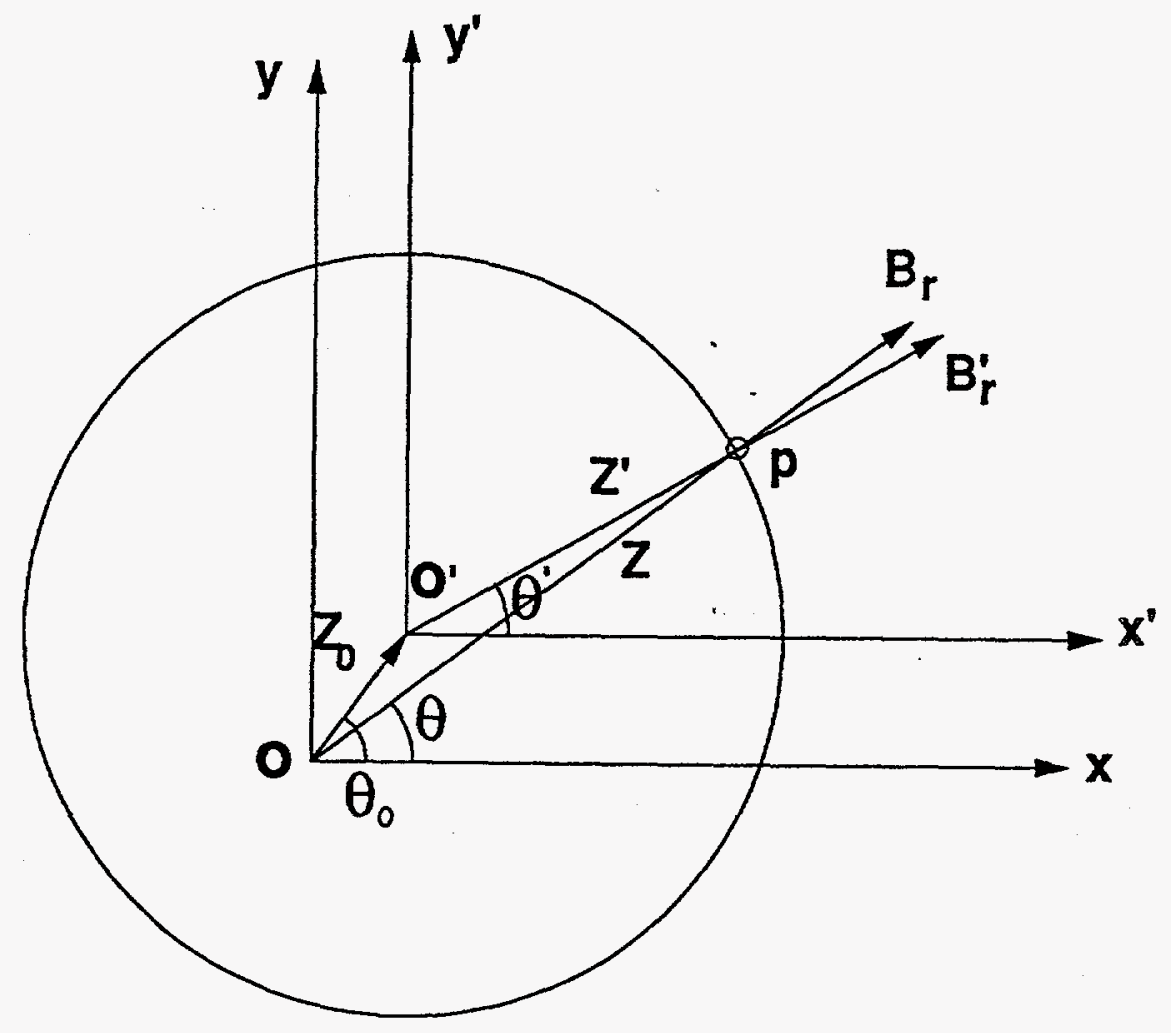

Fig.3. Coordinate systems of the magnetic center (MC) and cylinder rotation axis (CR). The MC is located at $O$. The $C R$, which is located at $O^{\prime}$,is displaced from $M C$ by $Z_{0}=r_{0} \exp \left(i \theta_{0}\right)$. The corresponding axes of the $x y$ and $x^{\prime} y^{\prime}$ coordinates are in parallel. $Z$ and $Z^{\prime}$ are coordinates of point $P$ on the cylinder surface with respect to the two coordinate systems. 


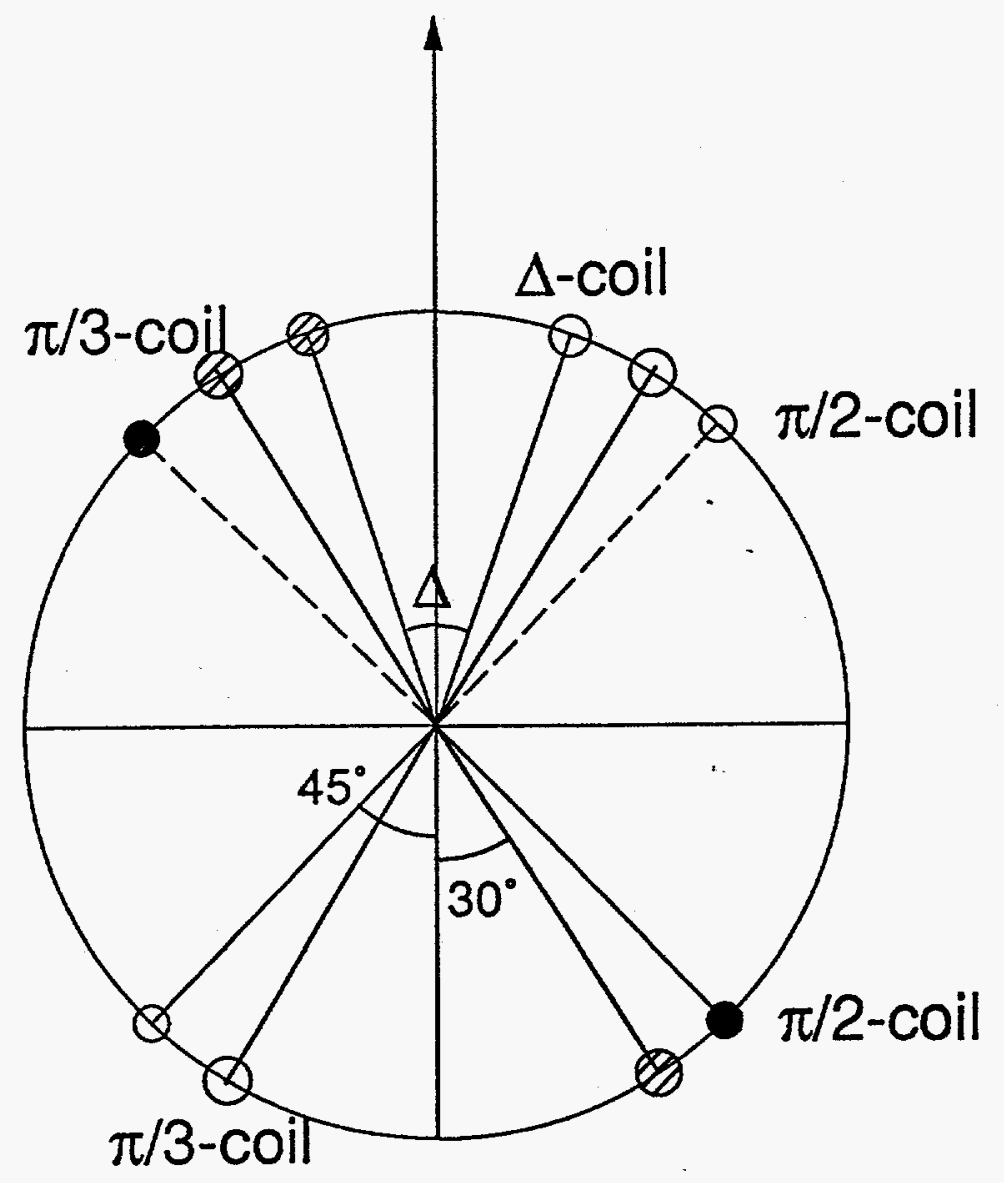

Fig.4. Cross section of a tangential winding coil for sextupole magnet measurements. It consists of one $\Delta$-coil, two $\pi / 2$-coils which is used for the bucking of quadrupole field and for the measurements of the magnetic center, and two $\pi / 3$-coils which is used for the bucking of the sextupole field and for the measurements of the sextupole field integral. 